\title{
COMPARACIÓN DE LA EFICACIA DE TRES MEDIOS DE CULTIVO in vitro PARA EL DESARROLLO DE Blastocystis spp
}

\author{
Comparison of the Efficacy of Three in vitro Media Culture for \\ BLASTOCYSTIS SPP
}

\author{
Linda Gallegos ${ }^{1}$, Armando González ${ }^{2,4}$, Teresa López-Urbina ${ }^{1}$, Eloy Gonzales- \\ Gustavson $^{3}$, Luis Gómez-Puerta ${ }^{2}$, Gianfranco Arroyo ${ }^{2}$
}

\section{Resumen}

\begin{abstract}
Blastocystis es un cromista que puede ser hallado en el tracto intestinal de animales y humanos y que ha pasado a ser considerado un patógeno emergente y de potencial zoonótico. El objetivo del trabajo fue comparar la eficacia de tres medios de cultivo in vitro, considerados como apropiados para el desarrollo del Blastocystis: Jones, Pavlova y Boeck-Drbohlav modificado (MBDM). Se utilizaron 54 muestras de heces de cerdos diagnosticados como positivos a Blastocystis por el método de sedimentación espontánea. El número de microorganismos inoculados fue clasificado como alto, intermedio y bajo. Las muestras fueron cultivadas por duplicado en los tres medios y al cabo de $72 \mathrm{~h}$ de incubación se procedió al recuento de microrganismos con la cámara de Neubauer. Los resultados indicaron que el medio Jones fue el más eficiente, seguido de los medios Pavlova y MBDM; asimismo, se obtuvieron mejores resultados en el cultivo cuando el nivel de microrganismos fue bajo. La forma quística fue la más comúnmente observada en las muestras de heces, mientras que en los medios de cultivo fue la vacuolar, seguida de la forma granular.
\end{abstract}

Palabras clave: Blastocystis spp, cerdos, cultivo in vitro, inóculo

\section{Abstract}

Blastocystis is a chromist that can be found in the intestinal tract of animals and humans, and is currently considered an emerging pathogen with zoonotic potential. The aim of this study was to compare the efficacy of three in vitro media culture which are considered appropriate for the development of Blastocystis: Jones, Pavlova and Boeck-

\footnotetext{
${ }^{1}$ Laboratorio de Microbiología y Parasitología Veterinaria, ${ }^{2}$ Laboratorio de Medicina Veterinaria Preventiva, Facultad de Medicina Veterinaria, Universidad Nacional Mayor de San Marcos, Lima

${ }^{3}$ Estación Experimental del Centro de Investigación IVITA, Universidad Nacional Mayor de San Marcos, El Mantaro, Huancayo

${ }^{4}$ E-mail: agonzale@jhsph.edu
} 
Drbohlav modified (BDM). It was used 54 stool samples from Blastocystis positive pigs diagnosed by the spontaneous sedimentation technique. The number of microorganisms inoculated was divided in three categories: low, intermediate and high. Each sample was cultivated for duplicate in the three media and after 72 hours of incubation the pathogens were counted using the Neubauer chamber. Results showed that the Jones media was the more efficient, followed by Pavlova and BDM; moreover, better results were obtained when the level of microorganisms was low. The cystic form was the most commonly observed in stool samples, whereas in culture media was the vacuolar followed by the granular form.

Key words: Blastocystis spp, pigs, in vitro culture, inoculum

\section{INTRODUCCIÓN}

Blastocystis spp son enteropatógenos que habitan el tracto intestinal, tanto de animales como de seres humanos. Los animales silvestres y domésticos son considerados como reservorios (Abe et al., 2002). Este microorganismo no parece ser muy patógeno en animales (Tan, 2008), aunque en humanos ha pasado a ser considerado un patógeno emergente, después de muchos años de ser considerado como habitante normal de la flora intestinal (Tan et al., 2010).

La clasificación taxonómica de este microorganismo ha sido incierta por muchos años. A finales de los ochenta, mediante la secuenciación génica de la subunidad menor ribosomal del ARN (ssARNr) (Stensvold $e t$ $a l ., 2007)$, se demostró que debería ocupar un lugar dentro del Reino Chromista, en el que también se encuentran diatomeas, oomicetos y algas marrones (Johnson et al., 1989). De acuerdo a la nueva clasificación, Blastocystis no es un hongo o protozoo, como antes se asumía, siendo el primer cromista conocido que parasita al humano (Tan et al., 2002).

Microorganismos del género Blastocystis se han descrito desde los inicios del siglo veinte, pero recién en los últimos años se han hecho avances en el entendimiento de su biología. Debido a su naturaleza polimórfica y a la falta de estandarización de técnicas para su diagnóstico, existe confusión e interpretación errónea de los resultados (Tan et al., 2010). Además, continúan las controversias relacionadas a los ciclos de vida propuestos, mecanismos de transmisión y patogenicidad, así como sobre la naturaleza propia del microorganismo y su papel como patógeno primario (Tan, 2008).

Blastocystis muestra una marcada variabilidad morfológica, mide entre 5 a $40 \mu \mathrm{m}$, carece de pared celular, y contiene mitocondrias, aparato de Golgi, y retículo endoplásmico liso y rugoso. Las formas reconocidas del microorganismo son la forma vacuolar, granular, ameboide y quística (Tan et al., 2010). También se encuentran otras formas menos frecuentes, como células avacuoladas y multivacuoladas, aunque no se sabe con exactitud los fenómenos que propician la variabilidad en su morfología (Stenzel y Boreham, 1996). La forma vacuolar es la que abunda en los cultivos celulares (Zierdt, 1991), la forma granular es comúnmente vista en cultivos tratados con antibióticos (Stenzel y Boreham, 1996) y la forma quística se observa mayormente en muestras de heces (Chen et al., 1999).

Este enteropatógeno exhibe una gran diversidad genética, habiéndose identificado nueve subtipos (ST) o genotipos (Stensvold et al., 2007). El hombre puede ser hospedador de diferentes ST de mamíferos (subtipo 1), primates y cerdos (subtipo 2), roedores (subtipo 4), bovinos y cerdos (subtipo 5) y de 
aves (subtipos 6 y 7) (Noel et al., 2005). Las personas que laboran en estrecho contacto con animales tienen una probabilidad mayor de infección con Blastocystis. Esto ha sido demostrado en guardianes de zoológicos (Parkar et al., 2011) y trabajadores de granjas de cerdos y aves (Rajah Salim et al., 1999). Además, ha sido identificado en asociación con la enfermedad inflamatoria del intestino y el síndrome del colon irritable (IBS) (Giacometti et al., 1999), ambas enfermedades gastrointestinales crónicas, de causas desconocidas y comunes en países desarrollados.

Existen varios métodos para la detección de Blastocystis spp en muestras de heces, que incluyen técnicas de concentración (suspensiones salinas, concentración con formol-éter), de coloración (tinción de Romanowsky, coloración permanente de frotis fecales fijadas con solución de acetato de sodio-ácido acético-formol), cultivo in vitro (Suresh y Smith, 2004), además de extracción y amplificación de ADN mediante una reacción en cadena de la polimerasa (PCR) (Stenzel y Boreham, 1996). No obstante, son los cultivos los considerados como «Gold Standard» para su detección ya que proporcionan suficiente ADN para llevar a cabo una tipificación molecular (Menounos et al., 2008).

Blastocystis spp pueden obtenerse de cultivos bifásicos o monofásicos (Clark y Diamond, 2002). Entre los medios idóneos para este enteropatógeno está el medio bifásico de Boeck-Drbohlav (MBDM), que contiene una fase líquida constituida por una solución tamponada y suero inactivado (bovino, caballo, conejo o humano (Zierdt, 1991; Lanuza et al., 1997). El medio de Jones es un medio monofásico ampliamente utilizado por su relativa simplicidad en la preparación y exigencias mínimas de mantenimiento (Jones, 1946; Suresh y Smith, 2004), y el medio de Pavlova, otro medio monofásico, descrito por algunos autores como de elección (De Martinez et al., 1996; INS, 2003; Zerpa et al., 2000), aunque sus ventajas com- parativas no han sido claramente demostradas.

Debido a la importancia que ha cobrado el Blastocystis como patógeno emergente, se están desarrollando distintas líneas de investigación, donde se requiere disponer de un gran número de estos microorganismos, ya sea para el desarrollo de modelos de infección experimental, preparación de antígenos para pruebas inmunodiagnósticas y elaboración de vacunas, así como para el desarrollo de pruebas moleculares de diagnóstico y genotipificación. El objetivo del presente estudio fue comparar la eficacia de tres medios de cultivo in vitro para el desarrollo de Blastocystis spp.

\section{Materiales y Métodos}

\section{Material Biológico}

Se utilizaron 54 muestras de heces positivas a Blastocystis spp, colectadas en abril de 2010 de cerdos criados en traspatio en el departamento de Tumbes, Perú, como parte de otro estudio cuyo objetivo fue evaluar la prevalencia de enteropatógenos y de cisticercosis porcina. Las muestras se trasladaron en refrigeración a la Facultad de Medicina Veterinaria de la Universidad Nacional Mayor de San Marcos, Lima.

Las muestras positivas a Blastocystis fueron identificadas con la prueba de sedimentación espontánea, siguiendo el protocolo descrito por Pajuelo-Camacho et al. (2006). Se seleccionaron las muestras que resultaron positivas a cualquiera de las formas reconocidas del microorganismo (vacuolar, granular, ameboide y quística), según las descripciones hechas por Tan et al. (2010). Los sedimentos se colocaron en tubos de $2 \mathrm{ml}$ y se almacenaron a $4{ }^{\circ} \mathrm{C}$. A los 60 días, las muestras se homogenizaron y se diluyeron 1:10 con agua destilada, para luego cuantificar los organismos con una cámara de Neubauer $\left(\mathrm{N}^{\circ}\right.$ de Blastocystis $/ \mathrm{ml}=\mathrm{N}^{\circ}$ de células x 5 x Factor de dilución x 104). Las 
Cuadro 1. Número de inóculos de Blastocystis spp empleados, según los cantidades de microorganismos presentes

\begin{tabular}{lrccc}
\hline Inóculo & $\mathrm{N}^{\circ}$ & Media & Mínimo & Máximo \\
\hline Bajo & 29 & $0.53 \times 10^{6}$ & $0.25 \times 10^{6}$ & $0.75 \times 10^{6}$ \\
Intermedio & 19 & $1.13 \times 10^{6}$ & $0.88 \times 10^{6}$ & $1.50 \times 10^{6}$ \\
Alto & 6 & $2.14 \times 10^{6}$ & $1.75 \times 10^{6}$ & $2.50 \times 10^{6}$ \\
\hline
\end{tabular}

muestras fueron inmediatamente cultivadas en los tres medios. El recuento de Blastocystis spp al finalizar el cultivo se realizó siguiendo las mismas direcciones que para la cuantificación del inóculo a partir de los sedimentos.

\section{Medios de Cultivo}

a) Medio Boeck-Drbohlav modificado $(M B D M)$. En la preparación de este medio, que consta de una fase líquida compuesta por la solución de Locke y otra sólida compuesta por la coagulación de huevos, se siguió el protocolo descrito por Lanuza et al. (1997).

b) Medio Pavlova. Se siguió el protocolo descrito en el Manual de Procedimientos para el Diagnóstico de Laboratorio de los Parásitos Intestinales del Hombre (INS, 2003).

c) Medio Jones. Se siguió el protocolo descrito por Jones (1946).

\section{Inoculación y Recuento}

Las muestras fueron homogenizadas con solución salina isotónica y se inocularon $500 \mu \mathrm{l}$, por duplicado, en cada uno de los medios de cultivo (MBDM, Pavlova y Jones). Se incubaron a $37{ }^{\circ} \mathrm{C}$ durante 72 horas, y luego se hizo el recuento de los microorganismos en la cámara de Neubauer, para lo cual se realizó una dilución de 1:10 con agua destilada, siguiendo el procedimiento descrito para las muestras de heces.

\section{Análisis de Datos}

El número de Blastocystis inoculados fue clasificado en tres categorías (bajo, intermedio y alto) para evaluar si la cantidad de microorganismos ejercía algún efecto sobre la cantidad de microorganismos resultantes. Los tres grupos fueron equidistantes entre sí $\left(<750 \times 10^{3}, 751 \times 10^{3}\right.$ a $1500 \times 10^{3}$ y $1501 \times 10^{3}$ a $\left.2500 \times 10^{3}\right)$.

Se utilizó la prueba de Shapiro Wilk para determinar si el recuento de microorganismos seguía la distribución normal, y en caso contrario, los datos serían transformados a logaritmo en base diez. Las diferencias entre medios de cultivo se determinaron mediante la prueba de análisis de varianza, utilizando el programa estadístico Stata $10 \AA$.

\section{Resultados}

Los resultados no seguían una distribución normal según la prueba de Shapiro Wilk $(\mathrm{z}=3.633, \mathrm{p}=0.0001)$. Los datos fueron transformados a logaritmo en base diez consiguiéndose una distribución normal $(\mathrm{z}=0.039$, $\mathrm{p}=0.5156$ ).

Se logró el crecimiento de Blastocystis en los tres medios de cultivos. La media del número de microorganismos inoculados fue de 0.92 x $10^{6}$ (IC 95\%: 0.84 x $10^{6}$ a 1.00 x $10^{6}$ ) y la media obtenida luego del cultivo fue de $3.34 \times 10^{6}$ (IC 95\%: $2.72 \times 10^{6}$ a $3.96 \times$ 


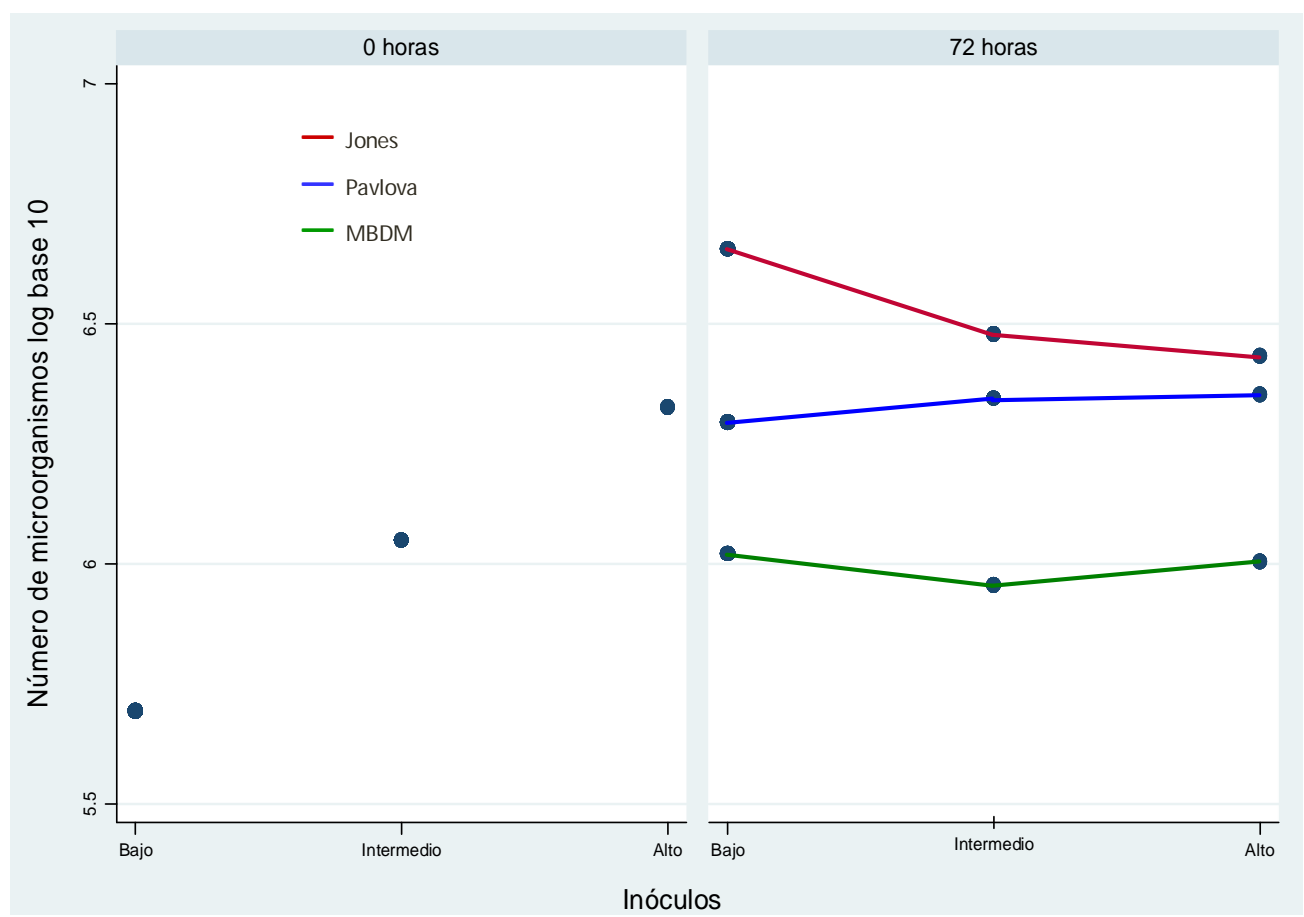

Figura 1. Distribución de las medias del número de Blastocystis spp transformados a logaritmo en base 10 antes y después del cultivo, y por medio de cultivo

$\left.10^{6}\right)$. En el Cuadro 1 se muestra el número de inóculos utilizados y las cantidades promedio de Blastocystis spp en las muestras de heces, según las tres categorías de concentración del enteropatógeno.

Las medias del número de microorganismos obtenidos por medio de cultivo y según el nivel de concentración se muestran en el Cuadro 2. Se encontró diferencia estadística entre medios $(F=20.55, p=0.000)$. Asimismo, se encontraron diferencias entre las categorías del inóculo $(\mathrm{F}=14.39, \mathrm{p}=0.000)$, obteniéndose los mejores resultados en el medio Jones cuando el número de microorganismos era más bajo (Fig. 1).

La forma de Blastocystis spp más frecuentemente observada en las muestras de heces fue la quística, generalmente sin membrana externa, mientras que en los medios de cultivos Jones y Pavlova fue la vacuolar (Fig. 2). La segunda forma más frecuentemente observada en las muestras cultivadas fue la granular, especialmente en el medio MBDM. Además, en los medios Jones y MDBD se encontraron quistes, tanto de membrana gruesa como de membrana delgada, aunque este hallazgo fue poco común.

\section{Discusión}

Los estudios iniciales realizados con cultivos de Blastocystis mostraban que este no crecía en medios que se utilizaban para cultivar amebas; sin embargo, se demostró que podía desarrollar en el medio MBDM y en el medio Jones, ambos utilizados para Entamoeba histolytica (Jones, 1946). En estos se utilizó una temperatura de $37^{\circ} \mathrm{C}, \mathrm{pH}$ entre 7.0 a 8.0 y la presencia de una flora bacteriana mixta, como Escherichia coli, Bacteroides spp, Streptococcus spp y Micrococcus spp, de allí que se pensara que Blastocystis poseía una naturaleza ameboide (Zierdt et al., 1967; Zierdt y Williams, 1973). 


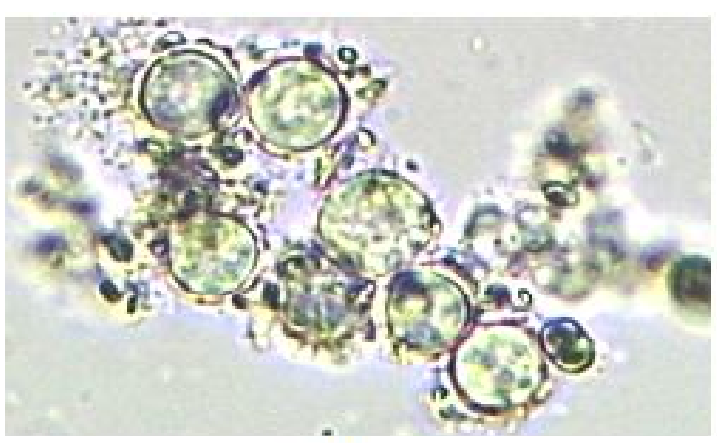

(A)

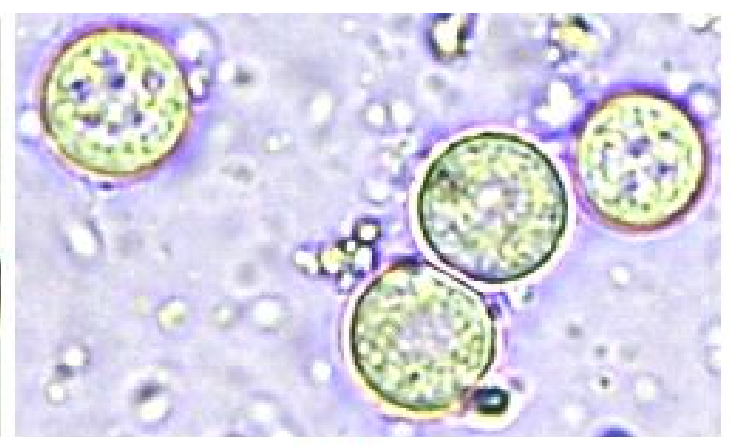

(B)

Figura 2. Blastocystis spp. (A) Formas vacuolares en medio Jones a las 72 horas (400X); (B) Formas granulares en medio MBDM a las 72 horas (400X)

El diagnóstico de infecciones por Blastocystis spp se basa usualmente en un examen microscópico de una extensión de heces o después de algún método de concentración. En este sentido, el cultivo in vitro puede incrementar la sensibilidad de tales métodos. Cuando se analizan muestras de heces, ya sea utilizando el medio Jones, extensiones fecales o concentración de heces con formalina-acetato de etilo, el cultivo en el medio Jones resulta ser el método más sensible en detectar Blastocystis (Leelayoova et al., 2002).

Cuando se compara el medio MBDM con microscopía de tinciones con hematoxilina férrica y con la prueba de PCR, los resultados no son tan eficientes como la prueba de PCR, pero supera con creces a la tinción hematoxilina férrica. Por tanto, se debe tener en cuenta que los medios de cultivos son necesarios para mejorar el diagnóstico y que la microscopía sola posee una baja sensibilidad cuando se usa como única modalidad de diagnóstico para la detección de Blastocystis spp (Roberts et al., 2011).

En el presente estudio se comparó el crecimiento in vitro de Blastocystis en los tres medios de cultivo más utilizados para su diagnóstico. El medio Jones resultó ser el mejor, en cuanto al número de microor- ganismos obtenidos, seguido del medio Pavlova. Asimismo, las formas vacuolares fueron las más frecuentes, seguido de formas granulares y en menor proporción de formas quísticas. Estos hallazgos concuerdan con otros estudios (Ho et al., 1993; Lanuza et al., 1997; Leelayoova et al., 2002; Roberts et al., 2011), los cuales reportan la forma vacuolar como predominante en los medios de cultivo.

Los resultados obtenidos en el presente trabajo con el medio Jones se atribuyen, probablemente, a que este medio no contiene antibióticos. Por otro lado, la concentración de antibióticos en el medio Pavlova fue menor que en el medio MBDM. Es posible que este factor haya inhibido a microorganismos como bacterias y levaduras necesarios en el metabolismo de nutrientes esenciales para el crecimiento de Blastocystis spp (Zierdt, 1991; Stenzel y Boreham, 1996).

Es probable que sustancias como el extracto de levadura y el almidón, presentes en los medios Jones y Pavlova, hayan influenciado como fuentes de nutrientes esenciales en el desarrollo del Blastocystis. El extracto de levadura proporciona nitrógeno orgánico, compuestos de carbono y elevadas concentraciones de vitaminas del complejo B (Tortota et al., 2007). Por otro lado, la pre- 
sencia del almidón es necesaria para el aprovechamiento del extracto de levadura, según se comprobó en el cultivo in vitro de Entamoeba hystolitica (Robinson, 1968).

Es frecuente que los medios de cultivo se complementen con suero animal entre $2 \mathrm{y}$ $20 \%$. El aporte del suero es fundamental para el desarrollo de los cultivos, dado que proporcionan factores de crecimiento y elementos mitogénicos, además de un ambiente muy estable para el crecimiento celular. El suero presenta, no obstante, ciertos inconvenientes, tales como la posible aparición de agentes contaminantes y la falta de homogeneidad del producto. En el presente estudio se trabajó con el medio suplementado con $5 \%$ de suero equino, siguiendo el protocolo original descrito por Jones (1946), aunque hay otros estudios que han utilizado el medio Jones suplementado con $10 \%$ de suero equino (Moe et $a l ., 1999)$, pero sin justificar su aumento proporcional.

Por lo observado en los resultados finales, se puede inferir que los microorganismos se reprodujeron con diferentes tasas de crecimiento, evidenciando que cantidades menores de microorganismos inoculados en un medio enriquecido pueden aprovechar con mayor eficiencia la mayor cantidad de nutrientes disponibles. Además, estudios llevados a cabo por Lanuza et al. (1997), describen la caída de la curva de crecimiento del Blastocystis a partir de las 72 horas, fenómeno que fue observado en el presente estudio.

El volumen en el medio Pavlova fue mayor que en los otros dos medios, lo cual aunado a la menor turbidez facilitaba el recuento de los microorganismos. En cambio, en el medio Jones, el cual presentó la mayor concentración de Blastocystis spp, se dificultó el recuento celular al microscopio. Por último, al momento de empezar el trabajo se necesitaba conocer el tiempo disponible para empezar el cultivo y se contaba con la información que estos microorganismos solo eran capaces de sobrevivir durante dos meses a temperatura de refrigeración (Yoshikawa et $a l ., 2004)$. Sin embargo, al final del estudio se pudo observar que los quistes permanecieron viables en las muestras de heces almacenadas a $4{ }^{\circ} \mathrm{C}$ durante más de nueve meses. Es muy probable que al ser cromistas, disponer de bacterias en su medio y estar en mayor proporción en su forma quística les haya permitido permanecer viables durante mucho tiempo.

\section{Conclusiones}

- El medio de cultivo más eficiente para Blastocystis spp fue el medio Jones.

- Se observó diferencia significativa en el recuento celular de los cultivos por efecto de la concentración inicial de Blastocystis spp en los inóculos $(\mathrm{p}<0.05)$.

- La forma celular más frecuente en las muestras de heces fue la quística, mientras que en los cultivos fue la forma vacuolar.

\section{Literatura Citada}

1. Abe N, Nagoshi M, Takami K, Sawano Y, Yoshikawa H. 2002. A survey of Blastocystis sp in livestock, pets, and zoo animals in Japan. Vet Parasitol 106: 203-212.

2. Chen XQ, Singh M, Howe J, Ho LC, Tan SW, Yap EH. 1999. In vitro encystation and excystation of Blastocystis ratti. Parasitology 118: 151-160.

3. Clark CG, Diamond LS. 2002. Methods for cultivation of luminal parasitic protists of clinical importance. Clin Microbiol Rev 15: 329-341.

4. De Martinez AM, Gomes MA, Viana Jda C, Romanha AJ, Silva EF. 1996. Isoenzyme profile as parameter to differentiate pathogenic strains of Entamoeba histolytica in Brazil. Rev Inst Med Trop Sao Paulo 38: 407-412. 
5. Giacometti A, Cirioni O, Fiorentini A, Fortuna M, Scalise G. 1999. Irritable bowel syndrome in patients with Blastocystis hominis infection. Eur $\mathrm{J}$ Clin Microbiol Infect Dis 18: 436-439.

6. Ho LC, Singh M, Suresh G Ng GC, Yap EH. 1993. Axenic culture of Blastocystis hominis in Iscove's modified Dulbecco's medium. Parasitol Res 79: 614-616.

7. [INS] Instituto Nacional de Salud. 2003. Manual de procedimientos de laboratorio para el diagnóstico de los parásitos intestinales del hombre. Lima: INS. Serie de manuales técnicos N. ${ }^{\circ} 37.90 \mathrm{p}$.

8. Johnson AM, Thanou A, Boreham PF, Baverstock PR. 1989. Blastocystis hominis: phylogenetic affinities determined by rRNA sequence comparison. Exp Parasitol 68: 283-288.

9. Jones WR. 1946. The experimental infection of rats with Entamoeba histolytica; with a method for evaluating the anti-amoebic properties of new compounds. Ann Trop Med Parasitol 40: 130-140.

10. Lanuza MD, Carbajal JA, Villar J, Borras $R$. 1997. Description of an improved method for Blastocystis hominis culture and axenization. Parasitol Res 83: 60-63.

11. Leelayoova S, Taamasri P, Rangsin $R$, Naaglor T, Thathaisong $U$, Mungthin M. 2002. In vitro cultivation: a sensitive method for detecting Blastocystis hominis. Ann Trop Med Parasitol 96: 803-807.

12. Menounos PG, Spanakos G Tegos N, Vassalos CM, Papadopoulou C, Vakalis NC. 2008. Direct detection of Blastocystis sp in human faecal samples and subtype assignment using single strand conformational polymorphism and sequencing. Mol Cell Probes 22: 24-29.

13. Moe KT, Singh M, Howe J, Ho LC, Tan SW, Chen XQ, Yap EH. 1999. Development of Blastocystis hominis cysts into vacuolar forms in vitro. Parasitol Res 85: 103-108.
14. Noel C, Dufernez F, Gerbod D, Edgcomb VP, Delgado-Viscogliosi P, Ho LC, et al. 2005. Molecular phylogenies of Blastocystis isolates from different hosts: implications for genetic diversity, identification of species, and zoonosis. J Clin Microbiol 43: 348-355.

15. Pajuelo-Camacho G, Luján-Roca D, Paredes-Pérez B, Tello-Casanova $R$. 2006. Aplicación de la técnica de sedimentación espontánea en tubo en el diagnóstico de parásitos intestinales. Ver Biomed 17: 96-101.

16. Parkar U, Traub RJ, Vitali S, Elliot A, Levecke B, Robertson I, Geurden T, et al. 2011. Molecular characterization of Blastocystis isolates from zoo animals and their animal-keepers. Vet Parasitol 169: 8-17.

17. Rajah Salim H, Suresh Kumar G, Vellayan S, Mak JW, Khairul Anuar A, Init I, Vennila GD, et al. 1999. Blastocystis in animal handlers. Parasitol Res 85: 1032-1033.

18. Roberts T, Barratt J, Harkness J, Ellis J, Stark D. 2011. Comparison of microscopy, culture, and conventional polymerase chain reaction for detection of Blastocystis sp in clinical stool samples. Am J Trop Med Hyg 84: 308-312.

19. Robinson GL. 1968. Laboratory cultivation of some human parasitic amoebae. J Gen Microbiol 53: 69-79.

20. Stensvold CR, Suresh GK, Tan KS, Thompson RC, Traub RJ, Viscogliosi E, et al. 2007. Terminology for Blastocystis subtypes - a consensus. Trends Parasitol 23: 93-96.

21. Stenzel DJ, Boreham PF. 1996. Blastocystis hominis revisited. Clin Microbiol Rev 9: 563-584.

22. Suresh K, Smith H. 2004. Comparison of methods for detecting Blastocystis hominis. Eur J Clin Microbiol Infect Dis 23: 509-511.

23. Tan KS. 2008. New insights on classification, identification, and clinical relevance of Blastocystis spp. Clin Microbiol Rev 21: 639-665. 
24. Tan KS, Singh M, Yap EH. 2002. Recent advances in Blastocystis hominis research: hot spots in terra incognita. Int J Parasitol 32: 789-804.

25. Tan KS, Mirza H, Teo JD, Wu B, Macary PA. 2010. Current views on the clinical relevance of Blastocystis spp. Curr Infect Dis Rep 12: 28-35.

26. Tortota G Funke B, Case C. 2007. Introducción a la microbiología. $9^{\circ}$ ed. Buenos Aires: Médica Panamericana. $959 \mathrm{p}$.

27. Yoshikawa H, Abe N, Wu Z. 2004. PCR-based identification of zoonotic isolates of Blastocystis from mammals and birds. Microbiology 150: 1147-1151.

28. Zerpa $R$, Huicho L, Náquira $C$, Espinoza I. 2000. A simplified culture method for Blastocystis hominis. Rev Mex Patol Clin 47: 17-19.

29. Zierdt CH, Rude WS, Bull BS. 1967. Protozoan characteristics of Blastocystis hominis. Am J Clin Pathol 48: 495-501.

30. Zierdt CH, Williams RL. 1973. Blastocystis hominis: axenic cultivation. Exp Parasitol 36: 233-243.

31. Zierdt CH. 1991. Blastocystis hominis- past and future. Clin Microbiol Rev 4: 61-79. 